\title{
WEIL REPRESENTATIONS AND CUSP FORMS ON UNITARY GROUPS
}

\author{
BY G. I. LEHRER
}

Communicated by Hyman Bass, March 22, 1974

Introduction. In this note we announce some results on the character theory of the finite unitary groups. The main result is the decomposition of the restriction of a certain class function $J$ to a maximal parabolic subgroup as a tensor product. This result is analogous with those of [4] for the general linear group $\operatorname{GL}(n, q)$, with the difference that the components of the tensor product are more complicated here. In fact, one has a Levi decomposition $P=M H$ of the maximal parabolic subgroup $P$, where $M$ is a unitary group of lower dimension, and $H$ is isomorphic to the "unitary Heisenberg group" (see below); one therefore (see [3], [6], [7]) obtains "Weil representations" of $P$, and these are instrumental in the decomposition of $J$.

Applications include an inductive proof that the restriction of $J$ to the unipotent subgroup of the unitary group is a proper character, and that $J$ has a nontrivial projection to the space of cusp forms on the unitary group in odd dimensions.

In some forthcoming work of Lusztig, the main theorem is used to show that $J$ is in fact a proper irreducible discrete series character of the unitary group, verifying a long-standing conjecture of Ennola [1]. ${ }^{1}$

1. The unitary group. We consider the finite unitary groups realized as follows: Let $G$ be $\operatorname{GL}\left(n, q^{2}\right)$, and consider the automorphism $\sigma$ of $G$ given by

$$
\sigma=-\circ-1 \circ t \circ Y,
$$

where $\circ$ denotes composition, - is conjugation (taking the matrix elements to the $q$ th power), $t$ is transposing of matrices and $Y$ is conjugation by the matrix

$$
\left[\begin{array}{llllll} 
& & & & & 1 \\
& 0 & & & 1 & \\
& & & \cdot & & \\
& & & & & 0 \\
1 & & & &
\end{array}\right] .
$$

AMS (MOS) subject classifications (1970). Primary 20G40, 20C15; Secondary 22E45.

${ }^{1}$ Added in proof. The author has recently learned that Ennola's conjecture for $J_{n}$ is still open. 
Then denote by $U\left(n, q^{2}\right)$, or $U_{n}$ when unambiguous, the group $\left\{g \in G \mid g^{\sigma}=g\right\}$.

The subgroup of $U_{n}$ with which we are concerned is in fact a subgroup of small index in a maximal parabolic subgroup. We do not take the whole parabolic subgroup to reduce the volume of unnecessary detail. The group $P$ we consider is that consisting of matrices $g$ in $U_{n}$ of the shape in Figure 1 .

$$
g=\left[\begin{array}{lll}
1 & \beta & x \\
0 & t & \alpha \\
0 & 0 & 1
\end{array}\right],
$$

FIGURE 1

where $t \in U_{n-2}, \alpha$ and $\beta \in V\left(n-2, q^{2}\right.$ ) (a vector space of dimension $n-2$ over $\left.\operatorname{GF}\left(q^{2}\right)\right)$ and $x \in \operatorname{GF}\left(q^{2}\right)$.

The condition $g^{\sigma}=g$ shows that $\beta$ is determined by $\alpha$, and $x$ satisfies $x+\bar{x}=-\langle\alpha, \alpha\rangle$, where $\langle$,$\rangle denotes the hermitian form on V\left(n-2, q^{2}\right)$ given by

$$
\left\langle\alpha, \alpha^{\prime}\right\rangle=\sum_{i=1}^{n-2} \alpha_{i} \bar{\alpha}_{n-1-i}^{\prime}
$$

We therefore denote $g$ above by $(t, \alpha, x)$.

The subgroup of $P$, consisting of $\{(t, \alpha, x) \mid \alpha=0, x=0\}$, is clearly isomorphic to $U_{n-2}$, and its normal complement $H_{n}=\{(t, \alpha, x) \mid t=I\}$ is called the unitary Heisenberg group. We have $P=U_{n-2} H_{n}$, the Levi decomposition.

2. The class function $J$. The Jordan canonical form shows that a conjugacy class in $\operatorname{GL}\left(n, q^{2}\right)$ is given by a partition-valued function on the set of irreducible polynomials over $\mathrm{GF}\left(q^{2}\right)$. Wall [5] has shown that two elements of $U\left(n, q^{2}\right)$ are conjugate if and only if they are conjugate in $\operatorname{GL}\left(n, q^{2}\right)$, and from this it is not hard to deduce that the conjugacy classes of $U_{n}$ are parametrized by partition-valued functions on the set of $U$-irreducible polynomials over $\mathrm{GF}\left(q^{2}\right)$ (see [1]), where a $U$-irreducible polynomial is either irreducible or a product of two related irreducible polynomials.

The work of Green [2] and subsequent authors showed that there is a family of distinguished characters of the general linear groups, called discrete series, which are in some sense necessary and sufficient for building the character table. In 1962, Ennola, by "twisting" the functions in Green's work, produced an orthonormal basis for the set of class functions on $U\left(n, q^{2}\right)$, which he conjectured were the irreducible characters of $U_{n}$. It is with the twisted form of a discrete series character of $\operatorname{GL}(n, q)$ that we are concerned. We define it simply by its values on the classes. 
Let $\psi$ be a fixed character of the multiplicative group $\operatorname{GF}\left(q^{2 n !}\right)^{*}$. Then define the class function $J=J_{n}$ by $J_{n}(c)=0$ unless $c=\left(f^{v}\right)(f$ being a $U$-irreducible polynomial), and

$$
J_{n}\left(f^{v}\right)=k\left(v,(-q)^{d(f)}\right) S_{d(f)}(\gamma)
$$

where: $p(v)$ is the number of parts of the partition $v$,

$$
k(v, t)=(1-t)\left(1-t^{2}\right) \cdots\left(1-t^{p(v)-1}\right),
$$

$d(f)=$ degree of the polynomial $f, \gamma$ is a root of $f$, and

$$
S_{d(f)}(\gamma)=\psi(\gamma)+\psi\left(\gamma^{-q}\right)+\psi\left(\gamma^{a^{2}}\right)+\cdots+\psi\left(\gamma^{(-\alpha)^{d(f)-1}}\right) .
$$

3. The Weil representations. The centre $Z$ of $H_{n}$ is $\{(t, \alpha, x) \mid t=I, \alpha=0\}$ and is isomorphic to the additive group $\mathrm{GF}(q)^{+} ; \boldsymbol{Z}$ is also the derived group of $H_{n}$. Hence $H_{n}$ has $\left|H_{n}\right| Z \mid=q^{2 n-4}$ distinct irreducible characters of degree one. It is easily seen further than $H_{n}$ has precisely $q-1$ other irreducible representations which are parametrized by the nontrivial characters $\chi$ of $\mathrm{GF}(q)^{+}$. We denote these by $\rho_{\chi}$, and their characters by $Q_{\chi}$. Now the group $U_{n-2}$ centralises the representation $\rho_{\chi}$, and we therefore obtain a projective representation $\tilde{\rho}_{x}$ (called a Weil representation) of $P=U_{n-2} H_{n}$, whose restriction to $H_{n}$ is $\rho_{\chi}$. Moreover we can prove

Proposition 3.1. (i) There is a representation $\tilde{\rho}_{x}$ of $P$ whose restriction to $H_{n}$ is $\rho_{\chi}$, and whose character $W_{\chi}$ takes rational values on unipotent elements.

(ii) The rationality condition in (i) determines $W_{\chi}$ uniquely on all unipotent subgroups of $P$.

The proof involves a characterisation of $\tilde{\rho}_{\chi}$ as an induced representation on unipotent subgroups, and the rationality comes from a consideration of the set of all $W_{\chi}$ as a whole.

In the determination of the values of $W_{\chi}$, the following lemma is of central importance (cf. Howe [3]).

Lemma 3.2. Suppose $V=V_{1} \perp V_{2}$ (where $V=V\left(n-2, q^{2}\right)$ and $\perp$ denotes the orthogonal direct sum with respect to $\langle\rangle$,$) , and let T$ be a unipotent group of unitary operators on $V$ such that $T V_{i}=V_{i}(i=1,2)$. Then if $H_{i}=\left\{(1, \alpha, x) \mid \alpha \in V_{i}\right\}$, and $W_{i}^{*}$ denotes the lift of the (unique) Weil character of $\mathrm{TH}_{i}$ to $\mathrm{TH}$, where $W_{i}$ corresponds to $\chi$, we have

$$
W_{\chi}=W_{1}^{*} \otimes W_{2}^{*} .
$$

4. The main theorem. Lemma 3.2 can be applied in various situations to give complete information about the values of $W_{\chi}$, which furnishes a proof of 
THEOREM A. Let $J_{n}$ be the function defined in $\S 2$ above, and let $J_{n-2}$ be the corresponding function (for the same $\psi$ ) on $U_{n-2}$. Then we have (for appropriate choice of $W_{\chi}$ )

$$
\begin{aligned}
J_{n} & =J_{n-2}^{P}-J_{n-2}^{*}+(-1)^{n-1} \sum_{\chi} J_{n-2}^{*} \otimes W_{\chi} \\
& =J_{n-2}^{*} \otimes \theta,
\end{aligned}
$$

where $J_{n-2}^{P}$ denotes the induced function, $J_{n-2}^{*}$ is the lift (or pullback) of $J_{n-2}$ from $U_{n-2}$ to $P$, and $\theta$ is the appropriate character of $P$ (given by the first line of the formula).

\section{Applications.}

COROLLARY 1. $J_{n}$ is a proper character (i.e. the character of a representation) of the maximal unipotent subgroup $S$ of $U_{n}$.

The proof is by induction on $n$ and depends on the observation that a copy of $S$ is contained in $P$, as the group of upper unitriangular matrices in $U_{n}$. This is because the automorphism $\sigma$ preserves the group of upper unitriangular matrices of $\operatorname{GL}\left(n, q^{2}\right)$. Theorem A applies equally well to $S$, where $W$ is interpreted as its-restriction to $S$ and so automatically gives a proof by induction, since $\theta$ is the character of a representation of $S$.

Corollary 2. For $n$ odd, $J_{n}$ has a nontrivial projection to the space of cusp forms on $U_{n}$.

The proof of this involves showing that certain scalar products are zeroviz. if $R$ is the radical of a maximal parabolic subgroup of $U_{n}$, it is required that $\left(J_{n}, I_{R}^{S}\right)=0$. Theorem $\mathrm{A}$, together with some general techniques of character theory, reduces the proof of this to the corresponding result for $J_{n-2}$.

As mentioned in the introduction, ${ }^{2}$ the work of Lusztig on the Brauer lifting of the natural representation of $U_{n}$ reduces the problem of showing that $J_{n}$ is a proper character of $U_{n}$ to Corollary 2 above, showing that here, $J_{n}$ is indeed a discrete series character. The generation of the whole character table of $U_{n}$ from the family of $J_{n}$ is complicated in this case, however, because the polynomials appearing in Ennola's table do not correspond to procedures such as inducing characters (i.e. are not structure constants in a Hall algebra) as is the case in $\operatorname{GL}(n, q)$.

Further details of this work shall appear in a forthcoming paper of the author.

\footnotetext{
${ }^{2}$ See footnote 1.
} 


\section{REFERENCES}

1. V. Ennola, On the characters of the finite unitary groups, Ann. Acad. Sci. Fenn Ser. AI No. 323 (1963), 35 pp. MR 28 \#143.

2. J. A. Green, The characters of the finite general linear groups, Trans. Amer. Math. Soc. 80 (1955), 402-447. MR 17, 345.

3. R. E. Howe, On the character of Weil's representation, Trans. Amer. Math. Soc. 177 (1973), 287-298.

4. G. I. Lehrer, Discrete series and the unipotent subgroup, Compositio Math. 28 (1974), 9-19.

5. G. E. Wall, On the conjugacy classes in the unitary symplectic and orthogonal groups, J. Austral. Math. Soc. 3 (1963), 1-62.

6. H. N. Ward, Representations of symplectic groups, J. Algebra 20 (1972), 182-195.

7. A. Weil, Sur certains groupes d'opérateurs unitaires, Acta Math. 111 (1964), 143-211. MR 29 \#2324.

Department of Pure Mathematics, University of Sydney, Sydney, New South Wales 2006, Australia 